\title{
A Conversion of Oral Cannabidiol to Delta9-Tetrahydrocannabinol Seems Not to Occur in Humans
}

\author{
Gerhard Nahler, ${ }^{1, *}$ Franjo Grotenhermen, ${ }^{2}$ Antonio Waldo Zuardi, ${ }^{3}$ and José A.S. Crippa ${ }^{3}$
}

\begin{abstract}
Cannabidiol (CBD), a major cannabinoid of hemp, does not bind to CB1 receptors and is therefore devoid of psychotomimetic properties. Under acidic conditions, CBD can be transformed to delta9-tetrahydrocannabinol (THC) and other cannabinoids. It has been argued that this may occur also after oral administration in humans. However, the experimental conversion of CBD to THC and delta8-THC in simulated gastric fluid (SGF) is a highly artificial approach that deviates significantly from physiological conditions in the stomach; therefore, SGF does not allow an extrapolation to in vivo conditions. Unsurprisingly, the conversion of oral CBD to THC and its metabolites has not been observed to occur in vivo, even after high doses of oral CBD. In addition, the typical spectrum of side effects of THC, or of the very similar synthetic cannabinoid nabilone, as listed in the official Summary of Product Characteristics (e.g., dizziness, euphoria/high, thinking abnormal/concentration difficulties, nausea, tachycardia) has not been observed after treatment with CBD in double-blind, randomized, controlled clinical trials. In conclusion, the conversion of CBD to THC in SGF seems to be an in vitro artifact.
\end{abstract}

Keywords: cannabidiol; delta9-tetrahydrocannabinol; acid-catalyzed cyclization; degradation; isomerization; gastric fluid

\section{Introduction}

Two recent publications of Merrick et al. ${ }^{1}$ and BonnMiller et al. ${ }^{2}$ have caused much confusion and uncertainty whether oral cannabidiol (CBD) is safe and whether subjects who are treated with CBD run the risk of positive workplace tests on delta9-tetrahydrocannabinol (delta9THC, in short THC) with the respective consequences. In this article, we would like to clarify a number of serious misinterpretations in the above mentioned articles and reinforce the arguments published recently. ${ }^{3}$

$\mathrm{CBD}$ and THC have a very similar chemical structure. Despite this similarity, they differ widely in their properties. CBD shows insufficient binding to the can- nabinoid receptors, particularly to $\mathrm{CB} 1$, which is involved in psychotomimetic effects. As to CB1, CBD is considered to be a negative allosteric modulator, which means, it modifies this receptor in such a way that the binding of classical agonists such as anandamide (AEA), THC, or nabilone is dramatically reduced. ${ }^{4}$ This effect is utilized also by Sativex ${ }^{\mathrm{TM}}$ to reduce some side effects of THC. Given that CBD is not a CB1 agonist, it is free from psychotomimetic properties and lacks cannabis-like intoxicating effects. This has been confirmed repeatedly by studies performed in the last four decades.

CBD, particularly in solution, is not fully stable; it needs to be stored at temperatures below $8^{\circ} \mathrm{C}$ and

${ }^{1} \mathrm{CIS}$ Clinical Investigation Support GmbH, Wien, Austria.

${ }^{2}$ Nova-Institut GmbH, Chemiepark Knapsack, Hürth, Germany.

${ }^{3}$ Department of Neuroscience and Behavior, University of São Paulo Ribeirão Preto, Brazil and Instituto Nacional de Ciência e Tecnologia Translacional em Medicina (INCT-TM).

*Address correspondence to: Gerhard Nahler, MD, PhD, CIS Clinical Investigation Support GmbH, Kaiserstr 43, 1070 Wien, Austria, E-mail: cis-qa@aon.at

(c) Gerhard Nahler et al. 2017; Published by Mary Ann Liebert, Inc. This is an Open Access article distributed under the terms of the Creative Commons Attribution License, which permits unrestricted use, distribution, and reproduction in any medium, provided the original work is properly cited. Mary Ann Liebert, Inc. offers reprint services for those who want to order professionally produced copies of articles published under the Creative Commons Attribution (CC BY) license. To obtain a price quote, email Reprints@liebertpub.com. Please include the article's title or DOI, quantity, and delivery destination in your email. 
protected from light. Under acidic conditions, CBD can be converted (isomerized) to THC and other cannabinoids. Therapeutically used $\mathrm{CBD}$ can be either plant derived, which is (-) trans CBD (purity $>99.5 \%$, CBD, e.g., of GW, United Kingdom or of BSPG, United Kingdom), or synthetic; the purity of marketed products is around $98 \%$ to $99 \%$ according to respective websites. Byproducts of plant-derived CBD are (-)cannabinoids, as the plant makes only one isomere, whereas impurities of synthetic CBD arise from remaining starting material and products formed during the synthesis. Traces of THC in less-purified CBD products cannot be excluded, but are unlikely of concern.

Based on in vitro conditions in simulated gastric fluid (SGF), it has been argued that this conversion of CBD to THC may also occur after oral administration. ${ }^{1,5}$ Such chemical transformations would not occur by other routes of administration, such as parenteral applications, particularly by inhalation, rectal, or transdermal application. In the following, the physiologic relevance of this in vitro transformation is discussed.

\section{SGF Does Not Reflect In Vivo Conditions}

Merrick et al. ${ }^{1}$ used an in vitro model with an SGF to study the conversion of CBD to delta-9THC and delta8-THC. This SGF was highly artificial; synthetic CBD (99\% purity) was dissolved in methanol and an aliquot diluted. The final SGF contained 1\% sodium dodecyl sulfate and $0.2 \%$ methanol, with a $\mathrm{pH}$ of 1 . There is no mention of the addition of electrolytes or gastric enzymes; overall test conditions deviate also from the U.S. Pharmacopoeia. In this assay, CBD degraded about $85 \%$ after $60 \mathrm{~min}$ and greater than $98 \%$ at 120 min, mainly to delta9-THC and delta8THC. Although the authors had cited a similar in vitro investigation by Watanabe et al., ${ }^{5}$ they did not discuss a striking discrepancy between these and their own results.

Watanabe et al..$^{5}$ incubated CBD of herbal origin (purity not stated) in modified artificial gastric juice without pepsin, but containing $\mathrm{NaCl}(2 \mathrm{mg} / \mathrm{mL}, \mathrm{pH}$ 1.2 ) at $37^{\circ} \mathrm{C}$. Even after $20 \mathrm{~h}$, the conversion rates for THC, cannabinol, 9alpha-hydroxyhexahydrocannabinol [ $9 \alpha-\mathrm{OH}-\mathrm{HHC}]$, and $8-\mathrm{OH}$-iso-HHC from CBD were only $2.9 \%, 1.1 \%, 1.4 \%$, and $10.0 \%$, respectively. This is a much lower conversion rate and strongly suggests that the composition of SGF and other test conditions has a major impact on the degradation of CBD.
The traditional medium to simulate gastric conditions in the fasted state has been SGF of the U.S. Pharmacopeial Convention (USP). This medium contains hydrochloric acid and sodium chloride, as well as pepsin and water, and has a $\mathrm{pH}$ of 1.2. Most often, it is prepared by dissolving $2.0 \mathrm{~g}$ of sodium chloride and $3.2 \mathrm{~g}$ of purified pepsin (derived from porcine stomach mucosa, with an activity of 800-2500 U per mg of protein) in $7.0 \mathrm{~mL}$ of hydrochloric acid and water up to $1000 \mathrm{~mL}$. However, it must be stressed that even this SGF significantly deviates from physiologic conditions. Most studies of gastric $\mathrm{pH}$ indicate that the across-the-board average gastric $\mathrm{pH}$ usually lies in the range $1.5-1.9 .^{6}$ To note, standard SGF is used to test the dissolution/disintegration of oral medications; it has not been developed to study chemical transformations. In fact, physiologic gastric juice is a very complex fluid with a $\mathrm{pH}$ around 1.5 to 1.9 to 3.5 (more acidic after a meal), and which contains a number of proteins to improve digestion, notably gastricsin, pepsin, trypsin (1 and 2), gastric lipase, gastric amylase, gelatinase, and mucin-glycoproteins, in addition to inorganic substances such as potassium, sodium, and calcium. ${ }^{7}$ Gastric transit time, although varying, is in the order of 2.5 to $3 \mathrm{~h}$ (for $50 \%$ of stomach contents emptied).

It is reminded that cannabinoids demonstrate significant protein binding; this could also protect CBD to some extent from a chemical transformation. ${ }^{8}$

As to the in vivo relevance of CBD degradation to THC and other cannabinoids, Watanabe et al. ${ }^{5}$ comment that "In biological systems, there have been no reports on the conversion of CBD to delta9-THC." We agree, despite intensive research, we are also unaware of any in vivo conversion of CBD to THC. In vivo, CBD undergoes extensive hydroxylation at multiple sites and further oxidations result in complex metabolic profiles; altogether, some 100 CBD metabolites have been identified. ${ }^{9}$ Compared to THC, the metabolism of CBD is unusually complex with considerable species variability.

\section{A Transformation of CBD to THC in the Stomach Should Demonstrate the Presence of Typical Metabolites of THC in Blood and Urine}

Detection of THC and metabolites in hair, urine and other body fluids is a well-established method in forensic laboratories worldwide. Merrick et al. write "even if just $1 \%$ of the CBD dose (i.e., $700 \mathrm{mg}$ ) were soluble, total cannabinoid levels, primarily delta9-THC and 
delta8-THC..., would be $6.5 \mathrm{mg}$ after $30 \mathrm{~min}$ and $13 \mathrm{mg}$ after $60 \mathrm{~min} . "$

11-Hydroxy- $\Delta$-tetrahydrocannabinol (11-OH-THC) is the primary, main psychoactive metabolite of delta9-tetrahydrocannabinol (THC). It is formed enzymatically by hydroxylation of the methyl side chain, predominantly by CYP2C9, and is the precursor of 11-nor-delta9-tetrahydrocannabinol-9-carboxylic acid (delta9-THC-COOH, also written as $\mathrm{THCCOOH}),{ }^{10}$ a main forensic marker. A test on $\mathrm{THCCOOH}$ is a sensitive method to prove the absorption of THC. THC $\mathrm{COOH}$ is not psychoactive. In urine, it is excreted mainly as glucuronide. This is important in view of the results observed by Harvey and Mechoulam. ${ }^{11}$

As the chemical structure of CBD differs to that of THC, the hydroxylation of the methyl side chain is predominantly catalyzed in vivo by a different enzyme, CYP2C19; mono-hydroxylated CBD metabolites are quickly formed, primarily 7-hydroxy-CBD (7-OH$\mathrm{CBD}$ ). Subsequently, 7-OH-CBD is oxidized to CBD7-oic acid, which is a major metabolite in both plasma and urine, and contains a hydroxyethyl side chain in addition. Free CBD, in large amounts, is excreted also in the feces. ${ }^{10}$ After repeated oral doses of $600 \mathrm{mg}$ $\mathrm{CBD} /$ day, Harvey and Mechoulam identified 33 metabolites in human urine in addition to unmetabolized $\mathrm{CBD}$ and several nonoxidized cyclized cannabinoids (see the interpretations by Harvey and Mechoulam, after next paragraph). ${ }^{11}$ If a transformation of $7-\mathrm{OH}$ $\mathrm{CBD}$ to psychoactive 11-OH-THC occurs in vivo, with the formation of a pyran ring (one oxygen atom and five carbon atoms), this should be early in this metabolization process, before the enzymatic hydroxylation. Although a test on 11-OH-THC is not specifically addressed in the publication of Harvey and Mechoulam, it seems to be unlikely that such an important metabolite would have been missed or not specifically mentioned.

An amount of 6.5 to $13 \mathrm{mg}$ THC is therapeutically effective and would produce plasma and urine tests that are positive on 11-OH-THC and THCCOOH., ${ }^{8,12}$ On the other hand, a much lower dose of $0.6 \mathrm{mg}$ THC (dissolved in hemp oil)/day over 10 days was found to produce no THCCOOH-positive tests in the urine of healthy volunteers. ${ }^{13}$ Therefore, if tests are negative on 11-OH-THC and particularly on THCCOOH, any dose of THC is likely in the order of $0.6 \mathrm{mg}$ THC or less.

To emphasize the in vivo relevance of their findings, Bonn-Miller et al. ${ }^{2}$ cite the above-mentioned publica- tion of Harvey and Mechoulam. ${ }^{11}$ However, not only were the amounts of delta8-THC and delta9-THC (benzopyran numbering) minimal $(1.97 \%$ and $0.69 \%$, respectively) but also, their glucuronide metabolites were not present in the urine as expected. Harvey and Mechoulam cautiously conclude that, ${ }^{11}$ "The formation of cyclized cannabinoids and, in particular, the THCs is also of interest. The mechanism for their formation is unknown and formation in the urine itself cannot be ruled out." One year later, they confirm this suspicion that, "The absence of metabolites of the THCs in the urine would suggest that cyclization had occurred after excretion."14

In short, according to Merrick et al., ${ }^{1}$ the majority of subjects receiving oral $\mathrm{CBD}$ would test positive on THC, with minimal amounts of CBD left ("In SGF, CBD degraded $\sim 85 \%$ after $60 \mathrm{~min}$ and greater than $98 \%$ at $120 \mathrm{~min}$ "). However, this conflicts with available data. There is no mentioning in the literature that typical THC metabolites had been found in blood or urine after oral CBD up to $800 \mathrm{mg}$, suggesting an in vivo transformation of CBD to THC. ${ }^{14,15}$ On the contrary, a significant percentage of CBD $(>25 \%)$ is excreted in urine unchanged or as glucuronide. ${ }^{11,14}$ Similarly, a recent double-blind, placebo-controlled crossover study that investigated the pharmacokinetics and safety of oral CBD (400 and $800 \mathrm{mg}$ ) coadministered with intravenous fentanyl found neither THC nor its main metabolites 11-OH-THC or THC-COOH in plasma and urine. ${ }^{16}$

Bonn-Miller et al. ${ }^{2}$ deny that the study of MartinSantos et al. ${ }^{17}$ demonstrates the absence of THC and its metabolites (i.e., 11-hydroxy-delta-9-tetrahydrocannabinol [11-OH-THC] or 11-nor-9-carboxy-delta-9-tetrahydro cannabinol [THC-COOH]) in whole blood (not in plasma as written by Bonn-Miller) after administration of CBD. There seems to be a serious misunderstanding upon the objectives of this study and therefore a misinterpretation of this graph. The study compared the pharmacokinetics and acute pharmacological effects after $10 \mathrm{mg}$ THC, $600 \mathrm{mg}$ CBD, and placebo in a crossover design. As is described in the text, whole blood levels of both, THC and CBD, are presented in the same graph. The authors write unambiguously that, "Levels of 11$\mathrm{OH}-\mathrm{THC}$ and $\mathrm{THCCOOH}$ were elevated after administration of THC (but not CBD or placebo) and followed a similar time course (fig. 6)." The legend of figure 6 perfectly corresponds "Time course of THC, 11-OH-THC, and THC-COOH whole blood levels after oral administration of $10 \mathrm{mg}$ of THC, $600 \mathrm{mg}$ of CBD, and placebo." 
Levels of THC and metabolites as given in this graph result from the administration of THC and not the degradation of CBD.

\section{A Transformation of CBD to THC Is Likely to Cause Typical Effects of THC}

Bonn-Miller et al. ${ }^{2}$ write "A number of the reviewed (and some not reviewed) studies documented somnolence, lethargy, fatigue, and poor motor and cognitive performance after administration of oral CBD, symptoms traditionally associated with THC," and cite publications that have already been included in the article of Merrick et al. ${ }^{1}$ A closer look on these publications reveals the following:

The study of Devinsky et al. ${ }^{18}$ (citation 13) was an open, "add-on" study; CBD was coadministered with standard epileptic medications. In such a design, adverse events caused by CBD cannot be separated clearly from those of comedications or those caused by interactions. This is only reliable with blinded, randomized, controlled clinical trials.

The review of Friedman and Devinsky. ${ }^{19}$ (citation 14) summarizes that, "Several studies suggest that CBD is nontoxic in nontransformed cells and does not induce changes on food intake, does not induce catalepsy, does not affect physiological parameters (heart rate, blood pressure, and body temperature), does not affect gastrointestinal transit, and does not alter psychomotor or psychological functions. Also, chronic use and high doses up to $1,500 \mathrm{mg}$ /day of CBD are reportedly well tolerated in humans." In fact, this is a citation out of the extensive review of Bergamaschi et al. ${ }^{20}$ on safety and side effects of CBD.

The study of Consroe et al. ${ }^{21}$ (citation 15) states the opposite of what is written by Bonn-Miller et al: "the inactivity of CBD, a major marijuana constituent, on motor and mental performance and effects also extends to its interaction with alcohol."

The study of Ramaekers et al. ${ }^{22}$ (citation 16) is a study on high-potency cannabis (13\% THC); thus it is not relevant for the properties of pure CBD.

Nicholson et al. ${ }^{23}$ (citation 17) summarize that, "Fifteen milligrams THC would appear to be sedative, while $15 \mathrm{mg}$ CBD appears to have alerting properties as it increased awake activity during sleep and counteracted the residual sedative activity of $15 \mathrm{mg}$ THC".

Obviously, this is again in conflict with the statements made by Bonn-Miller et al. ${ }^{2}$

For Marinol $^{\mathrm{TM}}$ (synthetic THC), the most frequent and typical side effects listed in the official Summary of Product Characteristics (www.drugs.com/pro/ marinol.html) are as follows ( $>3 \%)$ : abdominal pain, nausea, vomiting, dizziness, euphoria, paranoid reaction, somnolence, thinking abnormal, and furthermore ( $>1 \%$ to $3 \%$ ): asthenia, palpitations, tachycardia, vasodilation/facial flush, amnesia, anxiety/nervousness, ataxia, confusion, depersonalization, and hallucination. These are the principal reactions we would expect in case of a conversion of CBD to THC. It is reminded that the lowest observed adverse effect level (LOAEL) of oral THC has been estimated to $2.5 \mathrm{mg} /$ day. ${ }^{24} \mathrm{~A}$ THC dose of $6.5 \mathrm{mg}$ after $30 \mathrm{~min}$ and $13 \mathrm{mg}$ after $60 \mathrm{~min}$ as assumed by Merrick et al. would therefore be well above this threshold, likely causing typical adverse effects. Traces of THC are, however, unlikely of concern.

In the absence of any proofs for a conversion of CBD to THC in the stomach in vivo, the literature was searched for controlled clinical trials (most of them placebo controlled) with CBD to detect signs that could be interpreted, in view of the above, as typical THC reactions after oral administration of CBD. A brief look on some double-blind, randomized, parallel or cross-over patient trials shows that this spectrum of typical THC reactions has not been reported. ${ }^{25-32}$ These and additional studies have already been referred before. ${ }^{3}$ Although our list may still be incomplete, the conclusions of Bergamaschi et al. ${ }^{20}$ have been confirmed by other recent reviews. ${ }^{33,34}$

A very recent publication describing two cases of children with treatment-resistant epilepsy is of particular interest. Children first received CBD-enriched extracts that contained around $90 \%$ CBD in addition to 3-4\% D9-THC and standard antiepileptic therapy (case A, CBD dose $6 \mathrm{mg} / \mathrm{kg} /$ day; case B, $12.5 \mathrm{mg} / \mathrm{kg} /$ day). After 3 to 4 months of treatment, both children presented signs of intoxication by THC (inappropriate laughter/mild euphoria, ataxia, reduced attention, irritability, and eye redness, typical signs according to Martin-Santos et al). ${ }^{17}$ The CBD-enriched extracts (which remained the same during the initial treatment) were replaced by $200-300 \mathrm{mg} /$ day of purified CBD (purity $>99.6 \%$; BSPG, United Kingdom). This led to prompt and complete improvement of all intoxication signs. Follow-up assessments at 1 year (case A) and 1 year and 10 months (case B) showed remission of seizures and clear progressive improvement of the remaining general symptoms. ${ }^{35}$ Such evolution and lasting improvement could hardly be explained in case of a conversion of CBD to THC. 
Another clinical study, already cited above, concerning whole blood levels of THC and CBD, is also of interest for the pharmacological effects. In a randomized, double-blind, cross-over, placebo-controlled trial, healthy male subjects received oral THC $(10 \mathrm{mg})$ or CBD $(600 \mathrm{mg})$ or placebo. Relative to both placebo and $\mathrm{CBD}$, administration of THC was associated with anxiety, dysphoria, positive psychotic symptoms, physical and mental sedation, subjective intoxication effect at $2 \mathrm{~h}(p<0.01)$, and a significant increase in heart rate $(p<0.05)$. There were no differences between $\mathrm{CBD}$ and placebo on any symptomatic, physiological variable. $^{17}$

In conclusion, both publications, that of Merrick et al. ${ }^{1}$ and Bonn-Miller et al., ${ }^{2}$ are unfortunately misleading in many aspects. Over 40 years of research on $\mathrm{CBD}$ does not suggest a conversion of $\mathrm{CBD}$ to delta9-THC and/or other cannabinoids in vivo after oral administration. Such transformation occurs under artificial conditions, but is without any relevance for an oral therapy with CBD. Traces of delta9-THC in CBD per se, although theoretically possible in less-purified CBD productions, are unlikely to be of concern as long as the intake does not exceed the LOAEL.

\section{Acknowledgments}

J.A.C. and A.W.Z. are recipients of fellowship awards from Conselho Nacional de Desenvolvimento Científico e Tecnológico (CNPq, Brazil). This study is supported by a CNPq Grant (CNPq/MS/SCTIE/DECIT $\mathrm{N}^{\circ}$ 26/2014-Pesquisas sobre Distúrbios Neuropsiquiátricos; 466805/2014-4). BSPG-Pharm (Sandwich, United Kingdom) has kindly supplied CBD to our studies at no cost.

\section{Author Disclosure Statement}

G.N. is manager of an independent consultancy firm and medical advisor of Trigal Pharma GmbH. F.G. is Executive Director of IACM, www.cannabismed.org, and medical advisor of MedCann, www.medcann.de. A.W.Z. and J.A.C. are coinventors (Mechoulam R, JC, Guimaraes FS, AZ, JH, Breuer A) of the patent "Fluorinated CBD compounds, compositions and uses thereof. Pub. No.: WO/2014/108899. International Application No.: PCT/IL2014/050023"; Def. US No. Reg. 62193296; 29/07/2015; INPI em 19/08/ 2015 (BR1120150164927). University of São Paulo licensed it to Phytecs Pharm (Resolução USP No. 15.1.130002.1.1). University of São Paulo has an agree- ment with Prati-Donaduzzi (Toledo, Brazil): "Desenvolvimento de um produto farmacêutico contendo canabidiol sintético e comprovação de sua segurança e eficácia terapêutica na epilepsia, esquizofrenia, doença de Parkinson e transtornos de ansiedade." J.A.C. received a travel support from BSPG-Pharm.

\section{References}

1. Merrick J, Lane B, Sebree T, et al. Identification of psychoactive degradants of cannabidiol in simulated gastric and physiological fluid. Cannabis Cannabinoid Res. 2016;1:102-112.

2. Bonn-Miller MO, Banks SL, Sebree T. Conversion of cannabidiol following oral administration: authors' response to Grotenhermen et al. Cannabis Cannabinoid Res. 2017;2:5-7.

3. Grotenhermen F, Russo E, Zuardi AW. Even high doses of oral cannabidol do not cause THC-like effects in humans: Comment on Merrick et al. 2016 Cannabis Cannabinoid Res. 2017;2:1-4.

4. Laprairie RB, Bagher AM, Kelly ME, et al. Cannabidiol is a negative allosteric modulator of the type 1 cannabinoid receptor. Br J Pharmacol. 2015;172:4790-4805.

5. Watanabe K, Itokawa Y, Yamaori S, et al. Conversion of cannabidiol to D9tetrahydrocannabinol and related cannabinoids in artificial gastric juice, and their pharmacological effects in mice. Forensic Toxicol. 2007;25: $16-21$.

6. Klein S. The use of biorelevant dissolution media to forecast the in vivo performance of a drug. AAPS J. 2010;12:397-406.

7. Forte JG. Gastric function. In: Comprehensive Human Physiology (Greger R, Windhorst U, eds.). Springer Verlag: Berlin, 1996, pp. 1239-1257.

8. Huestis MA. Human cannabinoid pharmacokinetics. Chem Biodivers. 2007:4:1770-1804.

9. Ujvary I, Hanus L. Human metabolites of cannabidiol: a review on their formation, biological activity and relevance in therapy. Cannabis Cannabinoid Res. 2016;1:90-101.

10. Ujvary I, Grotenhermen F. 11-Nor-9-carboxy- $\Delta$ 9-tetrahydrocannabinol-a ubiquitous yet underresearched cannabinoid. A review of the literature. Cannabinoids. 2014;9:1-8.

11. Harvey DJ, Mechoulam R. Metabolites of cannabidiol identified in human urine. Xenobiotica. 1990;20:303-320.

12. Gustafson RA, Levine $B$, Stout PR, et al. Urinary cannabinoid detection times after controlled oral administration of D9-tetrahydrocannabinol to humans. Clin Chem. 2003:49:1114-1124.

13. Leson G, Pless P, Grotenhermen F, et al. Evaluating the impact of hemp food consumption on workplace drug tests. J Anal Toxicol. 2001;25: 691-698.

14. Harvey DJ, Samara E, Mechoulam R. Comparative metabolism of cannabidiol in dog, rat and man. Pharmacol Biochem Behav. 1991;40: 523-532.

15. Bergamaschi MM, Barnes A, Queiroz RHC, et al. Impact of enzymatic and alkaline hydrolysis on CBD concentration in urine. Anal Bioanal Chem. 2013:405:4679-4689.

16. Manini AF, Yiannoulos G, Bergamaschi MM, et al. Safety and pharmacokinetics of oral cannabidiol when administered concomitantly with intravenous fentanyl in humans. J Addict Med. 2015;9: 204-210.

17. Martin-Santos R, Crippa JA, Batalla A, et al. Acute effects of a single, oral dose of d9-tetrahydrocannabinol (THC) and cannabidiol (CBD) administration in healthy volunteers. Curr Pharm Design. 2012;18:4966-4979.

18. Devinsky O, Marsh E, Friedman D, et al. Cannabidiol in patients with treatment-resistant epilepsy: an open-label interventional trial. Lancet Neurol. 2016;15:270-278.

19. Friedman D, Devinsky O. Cannabinoids in the treatment of epilepsy. N Engl J Med. 2015;373:1048-1058.

20. Bergamaschi MM, Queiroz $\mathrm{RH}, \mathrm{Crippa} J A S$, et al. Safety and side effects of cannabidiol, a Cannabis sativa constituent. Curr Drug Safety. 2011;6: 237-249.

21. Consroe $P$, Carlini EA, Zwicker AP, et al. Interaction of cannabidiol and alcohol in humans. Psychopharmacology (Berl). 1979;66:45-50. 
22. Ramaekers JG, Kauert G, van Ruitenbeek $P$, et al. High-potency marijuana impairs executive function and inhibitory motor control. Neuropsychopharmacology. 2006;31:2296-2303.

23. Nicholson AN, Turner C, Stone BM, et al. Effect of delta-9tetrahydrocannabinol and cannabidiol on nocturnal sleep and earlymorning behavior in young adults. J Clin Psychopharmacol. 2004;24: 305-313.

24. EFSA, European Food Safety Authority. Scientific opinion on the risks for human health related to the presence of tetrahydrocannabinol (THC) in milk and other food of animal origin. EFSA J. 2015; 13:4141

25. Leweke FM, Piomelli D, Pahlisch F, et al. Cannabidiol enhances anandamide signalling and alleviates psychotic symptoms of schizophrenia. Transl Psychiatry. 2012;2:e94.

26. Morgan CJA, Freeman TP, Schafer GL, et al. Cannabidiol attenuates the appetitive effects of $\Delta$ 9-tetrahydrocannabinol in humans smoking their chosen cannabis. Neuropsychopharmacology. 2010;35: 1879-1885.

27. Jadoon KA, Ratcliffe SH, Barrett DA, et al. Efficacy cannabidiol and tetrahydrocannabivarin on glycemic and lipid parameters in patients with type 2 diabetes: a randomized, double-blind, placebo-controlled, parallel group pilot study. Diabetes Care. 2016;39:1-10.

28. Consroe P, Laguna J, Allender J, et al. Controlled clinical trial of cannabidiol in Huntington's disease. Pharmacol Biochem Behav. 1991;40: 701-708.

29. Chagas MH, Zuardi AW, Tumas V, et al. Effects of cannabidiol in the treatment of patients with Parkinson's disease: an exploratory doubleblind trial. J Psychopharmacol. 2014;28:1088-1098.

30. Notcutt W, Price M, Miller R, et al. Initial experiences with medicinal extracts of cannabis for chronic pain: results from 34 ' $\mathrm{N}$ of 1 ' studies. Anaesthesia. 2004:59:440-452.
31. Carlini E A, Cunha JM. Hypnotic and antiepileptic effects of cannabidiol. J Clin Pharmacol. 1981:21:417S-427S.

32. Wade DT, Robson $\mathrm{P}$, House $\mathrm{H}$, et al. A preliminary controlled study to determine whether whole-plant cannabis extracts can improve intractable neurogenic symptoms. Clin Rehabil. 2003;17:21-29.

33. Iffland K, Grotenhermen F. Safety and side effeccts of cannabidiol-a review of clinical data and relevant animal studies. European Industria Hemp Association. Available at: www.eiha.org (accessed January 4, 2017).

34. Fasinu PS, Phillips S, ElSohly MA, et al. Current status and prospects for cannabidiol preparations as new therapeutic agents. Pharmacotherapy. 2016;36:781-796.

35. Crippa JAS, Crippa ACS, Hallak JEC, et al. Delta 9-THC Intoxication by cannabidiol-enriched cannabis extract in two children with refractory epilepsy: full remission after switching to purified cannabidiol. Front Pharmacol. 2016:7:359.

Cite this article as: Nahler G, Grotenhermen F, Zuardi AW Crippa JAS (2017) A conversion of oral cannabidiol to delta9tetrahydrocannabinol seems not to occur in humans, Cannabis and Cannabinoid Research 2:1, 81-86, DOI: 10.1089/can.2017.0009.

\section{Abbreviations Used}

$\mathrm{CBD}=$ cannabidiol

$\mathrm{THC}=$ tetrahydrocannabinol

$\mathrm{SGF}=$ simulated gastric fluid

LOAEL $=$ lowest observed adverse effect level

\section{Publish in Cannabis and Cannabinoid Research}

Cannabis and

Cannabinoid

Research
- Immediate, unrestricted online access

- Rigorous peer review

- Compliance with open access mandates

- Authors retain copyright

- Highly indexed

- Targeted email marketing

liebertpub.com/can 